\title{
The Effect of Education on Compliance to a Workplace Health and Wellbeing Intervention: Closing the Loop
}

\author{
Lachlan Smith", Scott Pedersen, Dean Cooley \\ University of Tasmania, Faculty of Education, Active Work Laboratory, Launceston, 7248, TAS, Australia \\ *Corresponding Author: lqsmith@utas.edu.au
}

Copyright (C) 2013 Horizon Research Publishing All rights reserved.

\begin{abstract}
Desk-based worksites are increasingly the focus of workplace health and wellbeing interventions. These interventions often utilize an educational session prior to participants engaging, yet limited studies have specifically examined the effect education has on compliance. The purpose of this study was to investigate the effect pre-intervention participant education had on the odds of compliance to a passive e-health software program designed to increase non-exercise activity thermogenesis throughout the workday. Participants in the experimental group $(n=46)$ were exposed to multifaceted pre-intervention participant education one day prior to initiating the e-health software intervention for an eight-week study period. The control group $(n=33)$ also received the intervention for eight weeks, however these participants received no education prior to initiation. The e-health software had a self-report progress recording function, which recorded all logged movement entries to gain frequency counts of per day usage. The experimental group logged more active days, more movements per day and was significantly more compliant to the e-health software $(\mathrm{OR}=1.87,95 \% \mathrm{CI}=1.56-2.24)$. A pre-intervention participant education session, coupled with a passive e-health software program, was an effective mechanism for decreasing prolonged employee sedentary periods and increasing movement throughout the workday.
\end{abstract}

Keywords Workplace Health and Wellbeing, Pre-Intervention Participant Education, Prolonged Sitting

\section{Introduction}

Increasingly Australian workplaces are sites for health interventions. Similar to the rest of the world, the Australian population suffers from high rates of cardiovascular disease [CVD], which increases the risk of mortality and morbidity $[1,2]$. Sedentary behavior is considered a modifiable CVD risk factor [3] and voluntary exercise therapy is used to ameliorate this risk. Yet recent evidence suggests that prolonged sitting, which was once regarded as sedentary behavior, is a CVD risk factor independent of cardiovascular fitness $[3,4]$. Desk-based work has become more sedentary, leading to average sitting times of more than six hours for the full time Australian worker [5,6]. Moreover, changes to workplace environments and technology have lead to a decrease in non-exercise activity thermogenesis [NEAT] (>300 calories) for desk-based workers [7]. Thus this behavior represents a known health risk for desk-based workers. Only recently have health professionals targeted prolonged sitting in the workplace [8-10]. Almost all of these interventions include education as a principle component, yet the contribution of education to behavior change remains largely obscure for prolonged sitting. The aim of this study was to investigate the effect of pre-intervention participant education, coupled with a coping strategy based on principles of increasing NEAT, on employees' workplace behavioral health change.

Sedentary behavior has been characterized as low level or low amounts of physical activity [PA]. Recently, the current understanding of sedentary behaviors has broadened to include prolonged periods of sitting $[4,11,12]$. Prolonged sitting has been differentiated from sedentary behavior because long periods of inactivity result in different physiological effects on muscular biochemistry. For example, long periods of inactivity such as sitting for longer than four hours (cumulative) results in unique physiological changes, which include abnormal glucose metabolism [13], increased plasma glucose levels [14], and decreased lipoprotein lipase $[15,16]$. One way of reducing these outcomes is the prescription of PA. Although increasing voluntary PA seems to have little or no effect on ameliorating the risk posed by prolonged sitting $[17,18]$. Instead it appears that the use of regular short bursts of PA can reduce the CVD risk [19]. Since 2010, several studies have specifically investigated this potential strategy for reducing sedentary periods in the workplace $[8,10,20]$. One study on a workplace health and wellbeing intervention demonstrated that regular short bursts of PA ( $<10$ minutes) over the course of a workday reduced mean arterial blood pressure after 13 weeks [21]. Yet the authors did not disentangle whether it was the coping strategy or the raised threat level created through an education program, which contributed to the health behavior change. 
Behavior change can be influenced by multiple factors and therefore interventions need to be underpinned by theory. Health researchers have recently used protection motivation theory $[\mathrm{PMT}]$ to underpin the components of their health interventions [22]. PMT outlines the dual cognitive strategies of education and coping as a means of changing health behavior $[23,24]$. For example, education in the form of essays coupled with the coping strategies of eliminating sunbathing and using sunscreen resulted in an increase in individuals' healthy sun behaviors [22]. Furthermore, PMT messages have been used to test intentions for exercise behavior change to prevent CVD [24]. Thus, using PMT might be one way of assessing the impact of education on compliance to a health coping strategy in the workplace.

We used PMT as a framework to assess the effect of pre-intervention participant education on compliance to a workplace health and wellbeing intervention. The coping strategy in our project was not a typical workplace health and wellbeing intervention, because it involved an e-health desktop application based on a passive approach to participation during working hours. The passive approach involved a time generated prompt that forced employees' to engage in the intervention once an hour throughout the workday. Following other research designs [25-27] that have assessed the effect of pre-intervention participant education on compliance (e.g., medical advice and pamphlets), both the experimental and control group in this study received the passive coping strategy. Only the experimental groups' perceived threat associated with prolonged sitting was manipulated through the use of a pre-intervention participant education session. We hypothesized that employees who received pre-intervention participant education coupled with the coping strategy would comply significantly more to a workplace health and wellbeing intervention than participants who received only the coping strategy.

\section{Methods}

\subsection{Participants}

To compare the effect of education on compliance to a workplace health and wellbeing intervention, two state governmental agencies within Tasmania were purposefully selected based on a number of similar demographic parameters. For matching purposes all participants were desk-based employees who had sedentary working conditions due to their job responsibilities, which included administrative support, reception, and clerical responsibilities. Moreover, the workplace architecture within each agency had similar open plan worksites, shared office space with cubicles, and single offices.

All employees (approximately 200 desk-based workers) from these two agencies were invited to participate in the study via an intranet email from the respective occupational health and safety managers. This email introduced and explained the premise of the workplace health and wellbeing intervention and invited them to participate in an eight-week study to evaluate its effectiveness. Within this letter, participants were informed that the intervention was designed to prompt them to stand and move every hour in an effort to reduce prolonged sitting. The workplace health and wellbeing intervention was based on an e-health software called Exertime [8]. Exertime is a desktop application that prompts employees to remove themselves from a seated position every hour to engage in short bursts of PA throughout the workday and records this daily frequency. All employees in both agencies were informed that once the study was complete, everyone would have access to this software.

Initially, 107 desk-based employees volunteered to be a part of this study. A computer-based random numbers generator was used to select 80 participants from both of the agencies. A pilot study from our laboratory using a similar research design was able to elicit statistically significant differences with only 46 participants [8]. Therefore using a similar effect size we estimated that 80 participants, with the likelihood of a 10 per cent dropout rate, would provide sufficient power for our study.

Inclusion criteria were now applied to these randomly selected 80 participants. These participants were able to remain in the study if they were: (a) full time (five days a week) desk-based employees, (b) free from any debilitating health issues, and (c) using a desktop computer with internet access. Volunteers were asked to complete a PA readiness questionnaire [28] to ensure they were free from morbidity and neuromuscular conditions and able to participate in light intensity movements. One volunteer was excluded from participation due to health issues that prohibited this person from participating in a workplace health and wellbeing intervention. Thus, a sample of desk-based employees $(N=79)$ took part in an eight-week field-based, quasi-experimental intervention study.

Intact groups constrained by agency were randomly assigned to either receive the pre-intervention participant education coupled with the coping strategy (experimental group), or to only receive the coping strategy (control group). The experimental group $(n=46)$ had a gender disparity with $72 \%$ of participants being female $(n=33 ; \quad M$ age $=41.53+/-12.22)$ and $28 \%$ males $(n=13 ; \quad M$ age $=46.14+/-6.84)$. Participants in the control group $(n=33)$ were comparable with $70 \%$ of participants being female $(n=23 ; M$ age $=41.59+/-8.38 \mathrm{yr})$ and $30 \%$ males $(n=10 ; M$ age $=40.75+/-11.18$ ). Set inclusion criteria stated participants needed to work five days per week, however the field based nature of the study required flexibility for individuals missing days due to personal or sick leave among other reasons during the period of the study. Each participant in the sample provided informed consent in accordance with ethical guidelines set forth by the university.

\subsection{Experimental Group}


Participants in the experimental group $(n=46)$ received pre-intervention participant education one day before the e-health software was installed on their workplace desktop computers. The 50-minute pre-intervention participant education session, delivered by the research team, was held in a computer laboratory at a central training location for this agency. The research team had terminal degrees in the area of human movement and performance. Participants were exposed to face-to-face education that involved threat awareness associated with prolonged sitting coupled with the philosophy and directions on the use of the coping strategy. It also provided individual exposure to the coping strategy before implemented into the workplace. This allowed the experimental participants to ask the researchers questions about the threat and the coping strategy.

The multifaceted pre-intervention participant education session was divided into several key sections. Initially, 15 minutes were spent explaining the adverse health effects of prolonged sitting with particular emphasis placed on workplace sitting time. Charts were utilized to highlight research findings on CVD mortality rates among different job roles. Desk-based employees were advised that removing themselves from the seated position once every hour could produce a positive health effect. This advice was based on workplace guidelines [29] and recent health evidence [16]. The next 10 minutes were spent describing common difficulties associated with changing personal habits, relating specifically to breaking prolonged workplace sitting time and the benefits associated with modifying this habit. The focus shifted to the coping strategy with a 10 minute explanation and demonstration on how to perform PA within the workplace and examples of how to incorporate more movement into daily tasks. The final 10 minutes consisted of explicit instructions on how the desktop application will function and how to engage with it so that it is most effective. Participants were encouraged to try out the software and raise any questions or concerns in order to enhance their learning experience and understanding of the software.

Typical questions were centered on implementation and usage throughout the day (i.e., meetings, return from meetings), use in different work environments (i.e., home use, meeting use, video conferences), dose and frequency of movement (i.e., how many, intensity), and finally leave and absentee arrangements.

\subsection{Control Group}

Participants in the control group $(n=33)$ were from a separate matched government agency to ensure that there was no interaction with participants from the experimental group. The participants in this group also received an invitation email from their occupational health and safety manager appraising them of the workplace health and wellbeing intervention and research study to evaluate the program. The only further instructions participants in the control received were how to download and install the software on their desktop work computers. That is, the control group gained access to the intervention on the same day as the experimental group, without receiving pre-intervention participant education. Therefore, both groups had access to the intervention for a total of 40 workdays of full time employment.

\subsection{Data Collection}

Throughout the eight-week study, data were collected via the self-report progress recording function of the e-health software, which had two distinct phases. It utilized a set timed prompt in the form of a pop-up message on employees' computer screens that reminded them to break their seated position. The pop-up message provided two options, engage now or postpone. The postpone option was set to a maximum time of 60 minutes at which point the software locked the employee out of their computer forcing them to engage with the software. In the second phase, the software provided employees a choice of 60 office-appropriate movements (i.e., walking, stair climbs, chair squats) with the freedom to select the duration and intensity.

At the completion of each prompted sequence participants were required to $\log$ their movement engagement. That is, movements that required participants to $\log$ the number of repetitions they performed (e.g., 11 chair squats), the participants were prompted to enter these data in a text box. Alternatively, some movements required participants to time their engagement (e.g., walking: 30 seconds). For these movements the software contained a built-in clock and provided the recording for the participants. Each logged entry was date and time stamped by the software and transferred to a secure server so that compliance to the intervention could be monitored. The dependent variable to measure compliance was the number of logged entries tabulated over a workday. Each day that a participant logged seven or more movements (engaged with the intervention once an hour over an eight hour workday while taking a one hour lunch break) counted as a fully compliant day. Whereas a non-compliant day was measured as logging one to six movements during a workday.

\subsection{Data Analysis}

Data were entered into a Microsoft Excel database. Descriptive statistics of total days use and average daily use were described by means and standard deviations. Using commercially available software [30], odds within conditions (compliance/non-compliance), odds ratios [OR], and 95 per cent confidence intervals [CI] for the OR were generated using a 2 (compliance/non-compliance) $\mathrm{X} 2$ (education/no-education) contingency table. For each education condition, total number of days compliant (7 or more movements per day) and non-compliant (6 or less movements per day) for the eight-week experimental period were calculated and used as the frequency measure. A test of the research hypothesis was assessed using the 95 per cent CI for the OR. 


\section{Results}

All participants in the experimental group $(n=46)$ maintained the software on their desktop computers for the full eight weeks of the study. Across the 40-day period, participants in the experimental group logged more movements per day $(M=6.62 \pm 2.12)$ compared with participants in the control group $(M=5.22 \pm 1.53)$. The experimental group logged entries on more days $(M=29.93$ $\pm 8.37)$ and was fully compliant on more ( $>7$ movement) $(M$ $=17.1 \pm 10.9)$ of these days when compared to the control group with less total days $(M=22.36 \pm 9.84)$ and less compliant days $(M=9.3 \pm 7.87)$. An active day was defined for this study as a day when at least one movement was recorded on Exertime, which indicated that participants were at their desks at some point during the workday. The experimental group was active on 1377 days out of a possible $1840(75 \%)$ whereas the control group was active on 738 days out of a possible 1320 (56\%). As can be viewed in Table 1, the experimental group was compliant to the request on 786 days ( $57 \%$ of active days) while the control group was compliant on 307 days (42\% of active days).

Table 1. Total number of days for compliance and non-compliance in each education condition.

\begin{tabular}{cccc}
\hline & Group 1 & Group 2 & Total Days \\
\hline Compliance $\geq 7$ & 786 & 307 & 1093 \\
Non-compliance $<7$ & 591 & 431 & 1022 \\
Total Days & 1377 & 738 & 2115 \\
\hline
\end{tabular}

Group 1 = Experimental group, Group 2 = Control group

The results showed that odds of compliance were greater in the experimental group (Odds $=1.32$ ) compared to the control group (Odds=0.71). A test of the research hypothesis indicated when comparing the two groups, attending a pre-intervention participant education session significantly improved the odds of desk-based workers complying with the message to engage in some short burst PA every time the 45 minute prompt occurred compared to the control group $(\mathrm{OR}=1.87,95 \% \mathrm{CI}=1.56-2.24)$. Therefore, as the CI do not contain 1.00, participants who received pre-intervention participant education on the nature of the software operations and the health benefits of breaking periods of prolonged sitting every hour were 87 per cent more likely to comply to the software and engage in light movements on a regular basis throughout their workday compared to not having the knowledge prior to engaging.

\section{Discussion}

The findings from this study indicated that education coupled with a coping strategy was a superior combination, than just a coping strategy, for increasing compliance to a workplace health and wellbeing intervention. Employees in the experimental group demonstrated the positive effect pre-intervention participant education had on raising both threat and coping appraisal by recording more movements on more workdays during the eight-week trial. Our results are in congruence with those of previous studies that have utilized a PMT framework to investigate the influence education and coping strategies have on health behavioral change [22,24]. It appears that the influence of the two variables within this framework can be extended to elicit positive health behavior change in desk-based workers. This is encouraging given that the modifiable habit of prolonged sitting is a significant CVD risk factor, which is a major health issue for desk-based workers $[3,4]$. The pre-intervention participant education in this study might have raised employees' threat appraisal of prolonged sitting, leading to increased concerns for personal health and therefore increasing the likelihood of positive behavioral health change.

All participants in this study received the same coping strategy, a passive prompting e-health and wellbeing program called Exertime. The passive nature of the prompt created a situation in which employees were forced to engage with the program several times throughout the workday. Specific to this study was the vital role education played in enhancing the acceptance of the passive coping strategy. The passive approach might have caused frustration and aversion for participants who did not receive specific information on the functioning of the intervention. While the results clearly indicated the importance of pre-intervention participant education, this one session contained several educational strategies (threat awareness, potential coping strategies, education about the intervention, experiential learning). Thus, it is unclear which of these strategies or combination of strategies had the greatest impact on increasing compliance. Future investigations should consider disentangling the effect each of these strategies had on compliance.

Overall, our results suggested that desk-based employees were receptive to the idea of incorporating movement into their workday. In terms of our compliance analysis utilizing the criteria of seven movements per workday the experimental group was significantly better than the control group. Nonetheless, we should not overlook the level of compliance to the coping strategy exhibited by the control group. The workplace health and wellbeing intervention utilized in this study was founded on the relatively new idea of increasing NEAT in the lives of working adults with the purpose of improving their health [7]. Many participants in our study commented that they were unaware that the Exertime activities performed in short bouts throughout the workday could contribute to improving their health. This anecdotal finding suggested that desk-based employees embraced NEAT as a healthy alternative for energy expenditure. Interestingly, the concept of NEAT was only introduced to participants in the experimental group, yet the majority of participants from both groups were accepting of the coping strategy. This highlights the positive influence a workplace health and wellbeing intervention utilizing a passive approach can have on initiating and maintaining 
health behavior change. Although this trial was only eight weeks the majority of participants actively participated in the program for the duration of the study. Difficulties faced by health professionals in establishing new behaviors are documented in the literature [31], with expected time periods varying between 18 and 254 days for new health behaviors to become autonomous. This study demonstrated how an e-health and wellbeing program might be an achievable mechanism to promote sustainable change in desk-based employees' health behavior.

The results of this study had several possible implications. Educating people on the benefits of complying with health recommendations had the potential to reduce mortality and morbidity associated with CVD. However, to increase the likelihood of accepting the passive prompt our data suggests that education should explicitly outline and explain the coping strategy, discuss issues surrounding health behavior change, and provide threat awareness. Moreover, employees should have an opportunity to trial the program before initiation. Nonetheless, there were several limitations to this study. First, although we attempted to match our experimental and control groups this was only accomplished at the group level. Individuals within each group were not matched on demographic variables. Future research should attempt to address this limitation to manage non-intentional bias. We suggest that individuals be categorized using a stages of change inventory [32] to identify participants exercise behaviors and intentions within and outside of work. Second, the measure of compliance was based on self-report, which poses a possible threat to internal validity. The progress recording function of the software might not have provided an accurate measure of movements completed because participants had the opportunity to $\log$ a false movement driven by the desirability to be seen as active and committed to their health. We acknowledge the limitations of self-report [33], nonetheless, due to both groups of volunteers using the same self-reporting mechanism we believed that this nuance did not favour either group. Follow-up studies should be performed using an accurate objective measure, such as accelerometers $[10,20]$. Nonetheless, our results should be viewed with some caution as no objective measure was used in this study. Third, there might have been differences in the level of support employees received within each of the two government agencies in regards to participation in the workplace health and wellbeing intervention. For example, the level of endorsement by a supervisor might have varied between worksites. A final possible limiting factor of this research was the variable absenteeism noted between the two groups. This was evident by the experimental group having 19 per cent more active days than the control group. Due to the field-based nature of this study we were unable to account for all non-active days due to absenteeism, scheduled days off, professional development days, or days working at other worksites. While this field-based design might have contributed to the generalizability of our findings, future investigations should make note of these limitations. Perhaps a more accurate method to measure compliance, such as using accelerometers, will allow for comparisons with the self-reported data in this study to assess reliability and validity. Moreover, follow-up studies should examine any lasting behavioral change from participation in an eight-week period.

\section{Take Home Message}

Changing health behavior in the workplace, during work hours is a viable strategy to improve the health and wellbeing of working adults. Health professionals should provide education to raise threat awareness and explain coping strategies in order to increase the odds of participants complying with an e-health and wellbeing intervention based on a passive approach to elicit behavior change. The benefit of carefully planned pre-intervention participant education is an often underutilized and overlooked component of workplace health and wellbeing interventions. Future interventions aiming to reduce prolonged sitting time within the workplace should consider the efficacy of utilizing multifaceted pre-intervention participant education to increase the likelihood of behavior change.

\section{REFERENCES}

[1] P. T. Katzmarzyk, T. S. Church, C. L. Craig, C. Bouchard. Sitting time and mortality from all causes, cardiovascular disease, and cancer, Medicine \& Science in Sports \& Exercise, Vol. 41, No. 5, 998-1005, 2009.

[2] G. N. Healy, D. W. Dunstan, J. Salmon, E. Cerin, J. E. Shaw, P. Z. Zimmet, N. Owen. Breaks in sedentary time: Beneficial associations with metabolic risk, Diabetes Care, Vol. 31, No. 4, 661-666, 2008.

[3] M. T. Hamilton, G. N. Healy, D. W. Dunstan, T. W. Zderic, N. Owen. Too little exercise and too much sitting: Inactivity physiology and the need for new recommendations on sedentary behavior, Current Cardiovascular Risk Reports, Vol. 2, No. 4, 292-298, 2008.

[4] M. T. Hamilton, D. G. Hamilton, T. W. Zderic. Role of low energy expenditure and sitting in obesity, metabolic syndrome, type 2 diabetes, and cardiovascular disease, Diabetes, Vol. 56, No. 11, 2655-2667, 2007.

[5] A. Thorp, D. Dunstan, B. Clark, P. Gardiner, G. Healy, T. Keegel, N. Owen, E. Winkler. Stand up Australia: Sedentary behaviour in workers. Baker IDI Heart and Diabetes Institute, Cancer Prevention Research Centre at the University of Queensland, Medibank Private. Brisbane, QLD: Medibank Private Limited, 2009.

[6] A. A. Thorp, G. N. Healy, E. Winkler, B. K. Clark, P. A. Gardiner, N. Owen, D. W. Dunstan. Prolonged sedentary time and physical activity in workplace and non-work contexts: A cross-sectional study of office, customer service and call centre employees, International Journal of Behavioural Nutrition and Physical Activity, Vol. 128, 1-9, 2012.

[7] J. A. Levine, J. M. Miller. The energy expenditure of using a "walk-and-work" desk for office workers with obesity, British Journal of Sports Medicine, Vol. 41, No. 9, 558-561, 
2007.

[8] P. D. Cooley, S. J. Pedersen. A pilot study of increasing nonpurposeful movement breaks at work as a means of reducing prolonged sitting, Journal of Environmental and Public Health, Vol. 2013, 2013. doi.org/10/1155/2013/12837 6

[9] S. J. Pedersen, C. Mainsbridge, P. D. Cooley. An e-health intervention designed to increase workday energy expenditure by reducing prolonged occupational sitting habits, Work, in press.

[10] G. N. Healy, E. G. Eakin, A. D. LaMontagne, N. Owen, E. A. Winkler, G. Wiesner, L. Gunning, M. Neuhaus, S. Lawler, B. S. Fjeldsoe, D. W. Dunstan. Reducing sitting time in office workers: Short-term efficacy of a multicomponent intervention, Preventive Medicine, Vol. 57, No. 1, 43-48, 2013.

[11] N. Owen, A. Bauman, W. Brown. Too much sitting: A novel and important predictor of chronic disease risk? British Journal of Sports Medicine, Vol. 43, No. 2, 81-83, 2009.

[12] N. Owen, G. N. Healy, C. E. Matthews, D. W. Dunstan, Too much sitting: The population-health science of sedentary behaviour, Exercise and Sport Sciences Reviews, Vol. 38, No. 3, 105-113, 2012.

[13] D. W. Dunstan, J. Salmon, N. Owen, T. Armstrong, P. Z. Zimmet, T. A. Welborn, A. J. Cameron, T. Dwyer, D. Jolley, J. E. Shaw. Physical activity and television viewing in relation to risk of undiagnosed abnormal glucose metabolism in adults, Diabetes Care, Vol. 27, No. 11, 2603-2609, 2004.

[14] D. W. Dunstan, J. Salmon, G. N. Healy, J. E. Shaw, D. Jolley, P. Z. Zimmet, N. Owen. Association of television viewing with fasting and 2-h post-challenge plasma glucose levels in adults without diagnosed diabetes, Diabetes Care, Vol. 30, No. 3, 516-522, 2007.

[15] L. Bey, M. T. Hamilton. Suppression of skeletal muscle lipoprotein lipase activity during physical inactivity: A molecular reason to maintain daily low-intensity activity, Journal of Physiology, Vol. 551, No. 2, 673-682, 2003.

[16] M. T. Hamilton, D. G. Hamilton, T. W. Zderic. Exercise physiology versus inactivity physiology: An essential concept for understanding lipoprotein lipase regulation, Exercise and Sport Sciences Reviews, Vol. 32, No. 4, 161-166, 2004.

[17] D. W. Dunstan, B. Howard, G. Healy, N. Owen. Too much sitting: A health hazard, Diabetes Research and Clinical Practice, Vol. 97, No. 3, 368-376, 2012.

[18] D. W. Dunstan, A. A. Thorp, G. N. Healy. Prolonged sitting: Is it a distinct coronary heart disease risk factor? Current Opinion in Cardiology, Vol. 26, No. 5, 412-419, 2011.

[19] N. L. Glazer, D. W. Lyass, D. W. Esligeretal, S. J. Blease, P. S. Freedson, J. M. Massaro, J. M. Murabito, R. S. Vasan. Sustained and shorter bouts of physical activity are related to cardiovascular health, Medicine \& Science in Sports \& Exercise, Vol. 45, No. 1, 109-115, 2013.

[20] R. R. Evans, H. O. Fawole, S. A. Sheriff, P. M. Dall, M. Grant, C. G. Ryan. Point-of-choice prompts to reduce sitting time at work: A randomized trial, American Journal of Preventive
Medicine, Vol. 43, No. 3, 293-297, 2012.

[21] C. P. Mainsbridge, S. J. Pedersen, P. D. Cooley, S. P. Fraser. The effect of a passive prompting e-health and wellbeing program on cardiovascular health, Preventative Medicine, Submitted 07/2013; In review.

[22] S. Prentice-Dunn, B. F. McMath, R. J. Cramer. Protection Motivation Theory and stages of change in sun protective behaviour, Journal of Health Psychology, Vol. 14, No. 2, 297-305, 2009.

[23] D. L. Floyd, S. Prentice-Dunn, R. W. Rogers. A meta-analysis of research on protection motivation theory, Journal of Applied Social Psychology, Vol. 30, No. 2, 407-429, 2000.

[24] R. C. Plotnikoff, N. Higginbotham. Protection Motivation Theory and exercise behaviour change for the prevention of heart disease in a high-risk, Australian representative community sample of adults, Psychology, Health \& Medicine, Vol. 7, No. 1, 87-98, 2002.

[25] F. C. Bull, K. Jamrozik. Advice on exercise from a family physician can help sedentary patients to become active, American Journal of Preventive Medicine, Vol. 15, No. 2, 85-94, 1998.

[26] F. Gallefoss, P. S. Bakke. How does patient education and self-management among asthmatics and patients with chronic obstructive pulmonary disease affect medication? American Journal of Respiratory and Critical Care Medicine, Vol. 160, No. 6, 2000-2005, 1999.

[27] J. Hill, H. Bird, S. Johnson. Effect of patient education on adherence to drug treatment for rheumatoid arthritis: a randomized controlled trial, Annals of Rheumatic Diseases, Vol. 60, 869-875, 2001.

[28] R. J. Shepard. PAR-Q, Canadian home fitness test and exercise screening alternatives, Journal of Sports Medicine and Physical Fitness, Vol. 5, 185-195, 1988.

[29] Worksafe Australia. Guidance note for the prevention of occupational overuse syndrome in keyboard employment. In: National, Occupational, Health, and, Safety, Commission, editors. Canberra, Australia: National Government Publishing Service; 1996.

[30] Medcalc Software. Medicalc, Version 12.7.0: Medicalc Software, Ostend, Belgium, 2013.

[31] P. Lally, C. H. M. van Jaarsveld, H. W. W. Potts, J. Wardle. How are habits formed: Modelling habit formation in the real world, European Journal of Social Psychology, Vol. 40, 998-1009, 2010.

[32] J. O. Prochaska, C. C. DiClemente, J. C. Norcross. In search of how people change: Applications to addictive behaviors. American Psychologist, Vol. 47, No. 9, 1102-1114.

[33] D. Chan. So why ask me? Are self-report data really that bad? In C. E. Lance \& R. J. Vandenberg (Eds.), Statistical and methodological myths and urban legends: Doctrine, verity, and fable in the organizational and social sciences, 309-336, New York, NY: Routledge, 2009 